\title{
Magnetic Resonance Imaging in the Acquired Demyelinating Disorders: A Pediatric Cohort Study
}

\author{
Santa Ignêz L. J. ${ }^{1}$, Silveira de Souza A. ${ }^{2}$, Amâncio A. P. R. L. ${ }^{1}$, Almeida J.V. ${ }^{1}$, Gamarano G. M. F. ${ }^{1}$, Costa A. A. \\ B. $\mathrm{P}^{1}$, Tovar-Moll F. ${ }^{2,3}$ and Saad T. R. D. ${ }^{1}$ \\ 1. Fernandes Figueira National Institute of Women, Child and Adolescent Health-IFF/FIOCRUZ, Rio de Janeiro 22250-020, Brazil \\ 2. D'Or Research and Teach Institute-IDOR, Rio de Janeiro 22081-100, Brazil \\ 3. Federal University of Rio de Janeiro-UFRJ, Rio de janeiro 21941-901, Brazil
}

\begin{abstract}
Objective: Describing the characteristics of the MRI (magnetic resonance imaging) of patients with ADD (acquired demyelinating disorder) followed in a specialized pediatric clinic. Methods: Descriptive and retrospective study of the MRIs of a pediatric ADD cohort. The included images were acquired in machines with 1.5 or $3 T$ magnets. Low-quality images were excluded. The radiological characteristics of the lesions were described using the 2010 Revised McDonald Diagnostic Criteria regarding localization, contrast enhancement and optic nerve alterations. Results: Twenty-three patients were included (55\% female). The mean age of the first clinical event was 7.7 years. Most common diagnosis was Clinically Isolated Syndrome (35\%), followed by Multiple Sclerosis (30\%), Neuromyelitis Optica (17\%) and Acute Disseminated Encephalomyelitis (17\%). Mean time elapsed until diagnosis was 1.8 years. Follow-up MRIs of ADEM patients showed complete or partial resolution of lesions; MS lesions were mostly localized in the brain and four patients had contrast enhancement of lesions in their last available MRI. All NMO (neuromyelitis optica) patients had extensive spinal lesions, and two had optic neuritis. All patients with CIS (clinically isolated syndrome) had focal spinal lesions and evolved with radiologic improvement. Conclusions: ADEM and CIS patients' MRIs showed lesion reduction, while MS and NMO patients developed new lesions during follow-up.
\end{abstract}

Key words: Magnetic resonance imaging, multiple sclerosis, neuromyelitis optica, acute disseminated encephalomyelitis, clinically isolated syndrome.

\section{Introduction}

The ADD (acquired demyelinating disorder) represents immunologically mediated inflammatory and neurodegenerative conditions of the CNS (central nervous system). They can be characterized by the dysrupture of the myelin sheath and axonal damage, secondary to the neuroinflammatory process.

The main symptom of ADD is MS (multiple sclerosis), ADEM (acute disseminated encephalomyelitis), NMO (neuromyelitis optica) and CIS (clinically isolated syndrome). All of these conditions can have similar clinical and radiological characteristics in the early disease course, making the differentiation of each specific entity a challenge, and

Corresponding author: Lúcio Santa Ignêz, M.D., research fields: neurology. occasionally an impossible one, after the first acute episode of demyelination [1].

The worldwide prevalence of MS in adults varies between 5 and 30 cases per 100,000 persons [2]. But in about $5 \%$ of the adult patients, the symptoms begin before the age of 16 years old and, in less than 1\%, before the age of $10[3,4]$. Several retrospective studies published between 1968 and 1990 have demonstrated that many adult patients with MS had begun to show clinical symptoms during adolescence [3].

MRI (magnetic resonance imaging) has become the mainstay of complementary exams in the ADD [5], playing a major role not only in diagnostics, but prognostics and follow-up. It is used in the definition of the pediatric ADD diagnostic criteria established in the 2007 international consensus [6], later revised in 
2013 [7]. The widespread access to MRI, in association to the refinement of neuroimaging techniques and deeper knowledge of the spectrum of these diseases lead to an increase in the number of diagnosed and reported cases. Even then, the ADD still represents a group of rare diseases in the pediatric population.

In Brazil there is a limited number of centers specialized in pediatric ADD. Our group was the first to be founded in the state of Rio de Janeiro, in 2012, and still remains the only one available for a population of over 16.5 million people. It is one of the very few pediatric hospitals that offer evaluation, treatment and multidisciplinary follow-up of ADD in patients under 18 years old, country-wide. This specialized take on patient care allows for a uniform diagnostic and therapeutic approach.

The main objective of this study is to describe the MRI characteristics of these patients. It is the first longitudinal and prospective study of an exclusively pediatric cohort followed by any single medical institution in Brazil.

\section{Materials and Methods}

Descriptive study of a bidirectional cohort of patients diagnosed with ADD under the age of 18 years old, followed longitudinally in a specialized pediatric ADD clinic. Patients with suspected ADD were referred to us for evaluation and all of those with confirmed ADD were included in the study.

The diagnosis of ADD subtypes was established in agreement with the definition proposed by the International MS Study Group of 2007 [6], revised in 2013 [7].

All patients underwent brain, orbits and spinal cord MRI using $1.5 \mathrm{~T}$ or $3 \mathrm{~T}$ magnetic fields. All images obtained, including the ones previous to admission to our clinic, were added in the study. Because images could not always be obtained in the same MRI machine, mainly due to patients' varied economical and health insurance related situations, some images did not meet minimal quality standards (due to movement artifacts, digitalization errors or those obtained using a magnetic field inferior to $1.5 \mathrm{~T}$ or those using an open magnet) and could not be adequately evaluated. These images were excluded from the study.

Twenty-seven patients were evaluated in total and they are listed in Table 1. Patients' number 21 and 24 did not fulfill the inclusion criteria, being diagnosed with conditions other than ADD (in one case, a post-infectious optic neuritis, and the other a post-vaccine transverse myelitis). Patients 8 and 10 were not included from the study because their imaging studies did not meet the quality standards required for evaluation. Patient's numbers on the table refer to their chronological order of entry in the study.

Demographic and clinical data collected from the patients included: sex, age at first demyelinating episode, age of admission at our clinic, neurological function in eave acute episode (according to the EDSS (expanded disability status scale)), disease duration, number of acute clinical events, relevant laboratory exams [OCBs (oligoclonal bands) and AQP4-IgG (anti-aquaporin-4 IgG antibody)] and the last ADD specific medication used by each patient.

The MRI exams were evaluated by a radiologist with expertise in pediatric neuroradiology, blind to the clinical data. It was used the McDonald criteria of 2010 [8], and the International Pediatric MS Study Group definitions of 2007 [6], revised in 2013 [7], observing lesions in regards to their localization (in the brain, considering periventricular, juxtacortical, infratentorial and corpus callosum involvement, and in the spinal cord, considering cervical, thoracic and lumbar involvement), longitudinal extension (focal if equal to or under 2 vertebral bodies, or extensive if over 3 vertebral bodies), contrast (gadolinium) enhancement and involvement of optic nerves (presence of hyperintense signal in FLAIR or T2-weighted images, swelling or reduced volume and contrast enhancement). 
Table 1 Clinical and laboratory data of patients with demyelinating diseases.

\begin{tabular}{|c|c|c|c|c|c|c|c|c|c|}
\hline Subjec & $\begin{array}{l}\text { Age at } 1^{\circ} \\
t \text { clinical event } \\
\text { (years) }\end{array}$ & Sex & $1^{\circ}$ clinical event & $\begin{array}{l}\text { urological functions } \\
\text { linical presentation } \\
2^{\circ} \text { clinical event }\end{array}$ & $3^{\circ}$ clinical event & $\begin{array}{l}\text { Final diagnosis/ } \\
\text { BOC/AQP4 }\end{array}$ & $\begin{array}{l}\begin{array}{l}\text { Age at } \\
\text { diagnosis } \\
\text { (years) }\end{array} \\
\end{array}$ & $\begin{array}{l}\text { Total disease } \\
\text { time } \\
\text { (years) }\end{array}$ & $\begin{array}{l}\text { Immunomodulatory or } \\
\text { immunosuppressive drugs in } \\
\text { use at the end of study }\end{array}$ \\
\hline 1 & 4 & $\mathrm{~F}$ & $\begin{array}{l}\text { Pyramidal, cerebral and } \\
\text { visual }\end{array}$ & $\begin{array}{l}\text { Pyramidal, brainstem } \\
\text { and visual }\end{array}$ & Brainstem & $\begin{array}{l}\text { ADEM (OCB not } \\
\text { performed) }\end{array}$ & 6 & 8.0 & Mycophenolate mofetil \\
\hline 2 & 4 & $\mathrm{~F}$ & $\begin{array}{l}\text { Pyramidal and bowel } \\
\text { and bladder }\end{array}$ & $\begin{array}{l}\text { Pyramidal and } \\
\text { cerebellar }\end{array}$ & $\begin{array}{l}\text { Pyramidal and } \\
\text { cerebellar }\end{array}$ & NMO (AQP4-IgG -) & 11 & 7.8 & Glatiramer acetate \\
\hline 3 & 1 & $\mathrm{~F}$ & Cerebellar & $\begin{array}{l}\text { Pyramidal and } \\
\text { cerebellar }\end{array}$ & Pyramidal & $\mathrm{MS}(\mathrm{OCB}+)$ & 2 & 6.8 & Interferon $\beta$ 1A $44 \mu \mathrm{g}$ \\
\hline 4 & 4 & M & Pyramidal and mental & & & $\begin{array}{l}\text { ADEM (OCB Not } \\
\text { performed) }\end{array}$ & 4 & 5.8 & Antiepileptic drug \\
\hline 5 & 5 & M & Brainstem & $\begin{array}{l}\text { Pyramidal, cerebellar } \\
\text { and brainstem }\end{array}$ & & $\mathrm{MS}(\mathrm{OCB}+)$ & 6 & 5.2 & Interferon $\beta 1 \mathrm{~A} 44 \mu \mathrm{g}$ \\
\hline 6 & 13 & M & Brainstem and visual & $\begin{array}{l}\text { Pyramidal and } \\
\text { cerebellar }\end{array}$ & $\begin{array}{l}\text { Pyramidal, } \\
\text { cerebellar and } \\
\text { visual }\end{array}$ & $\mathrm{MS}(\mathrm{OCB}+)$ & 14 & 5.8 & Interferon $\beta 1 \mathrm{~A} 44 \mu \mathrm{g}$ \\
\hline 7 & 13 & $\mathrm{~F}$ & Brainstem and visual & $\begin{array}{l}\text { Pyramidal and } \\
\text { cerebellar }\end{array}$ & & MS (OCB -) & 14 & 4.8 & Interferon $\beta 1 \mathrm{~A} 44 \mu \mathrm{g}$ \\
\hline $8 * *$ & --- & --- & --- & --- & --- & --- & --- & --- & --- \\
\hline 9 & 1 & $\mathrm{~F}$ & Brainstem and visual & $\begin{array}{l}\text { Pyramidal, cerebellar } \\
\text { and visual }\end{array}$ & Pyramidal & $\begin{array}{l}\text { NMO (AQP4-IgG } \\
+)\end{array}$ & 3 & 5.8 & $\begin{array}{l}\text { Azatioprine }+ \\
\text { Mycophenolate mofetil }\end{array}$ \\
\hline $10 * *$ & --- & --- & --- & --- & --- & --- & --- & --- & --- \\
\hline 11 & 7 & M & $\begin{array}{l}\text { Pyramidal, cerebral, } \\
\text { bowel and bladder }\end{array}$ & & & $\begin{array}{l}\text { ADEM (OCB not } \\
\text { performed) }\end{array}$ & 9 & 4.1 & Antiepileptic drug \\
\hline 12 & 8 & M & Visual & & & $\begin{array}{l}\text { CIS (OCB + } \\
\text { /AQP4-IgG -) }\end{array}$ & 8 & 3.4 & Without medication \\
\hline 13 & 11 & M & $\begin{array}{l}\text { Cerebellar, brainstem, } \\
\text { bowel and bladder }\end{array}$ & Pyramidal & & $\begin{array}{l}\text { MS (OCB not } \\
\text { performed) }\end{array}$ & 12 & 2.9 & Interferon $\beta 1 \mathrm{~A} 44 \mu \mathrm{g}$ \\
\hline 14 & 7 & M & $\begin{array}{l}\text { Pyramidal, cerebellar } \\
\text { and brainstem }\end{array}$ & & & CIS (OCB -) & 7 & 2.4 & Without medication \\
\hline 15 & 11 & $\mathrm{~F}$ & $\begin{array}{l}\text { Pyramidal, sensory, } \\
\text { visual, bowel and } \\
\text { bladder }\end{array}$ & & & NMO (AQP4-IgG -) & 11 & 2.3 & Glatiramer acetate \\
\hline 16 & 7 & $\mathrm{~F}$ & Cerebral & & & $\begin{array}{l}\text { ADEM (AQP4-IgG } \\
-)\end{array}$ & 7 & 2.3 & Antiepileptic drug \\
\hline 17 & 5 & $\mathrm{~F}$ & $\begin{array}{l}\text { Pyramidal, cerebellar } \\
\text { and cerebral }\end{array}$ & & & $\begin{array}{l}\text { CIS (OCB + } \\
\text { /AQP4-IgG -) }\end{array}$ & 12 & 8.3 & Without medication \\
\hline 18 & 9 & $\mathrm{~F}$ & Pyramidal & & & $\mathrm{MS}(\mathrm{OCB}+)$ & 11 & 2.3 & Interferon $\beta 1 \mathrm{~A} 44 \mu \mathrm{g}$ \\
\hline
\end{tabular}


(Table 1 continued)

\begin{tabular}{|c|c|c|c|c|c|c|c|c|c|}
\hline Subject & $\begin{array}{l}\text { Age at } 1^{\circ} \\
t \text { clinical event } \\
\text { (years) }\end{array}$ & Sex & $1^{\circ}$ clinical event & $\begin{array}{l}\text { eurological functions } \\
\text { Clinical presentation } \\
2^{\circ} \text { clinical event } \\
\end{array}$ & $3^{\circ}$ clinical event & $\begin{array}{l}\text { Final diagnosis/ } \\
\text { BOC/AQP4 }\end{array}$ & $\begin{array}{l}\text { Age at } \\
\text { diagnosis } \\
\text { (years) }\end{array}$ & $\begin{array}{l}\text { Total disease } \\
\text { time } \\
\text { (years) }\end{array}$ & $\begin{array}{l}\text { Immunomodulatory or } \\
\text { immunosuppressive drugs in } \\
\text { use at the end of study }\end{array}$ \\
\hline 19 & 12 & $\mathrm{~F}$ & $\begin{array}{l}\text { Pyramidal, sensory, } \\
\text { cerebral, bowel and } \\
\text { bladder }\end{array}$ & & & CIS (AQP4-IgG -) & 13 & 1.8 & Without medication \\
\hline 20 & 9 & M & $\begin{array}{l}\text { Pyramidal, bowel and } \\
\text { bladder }\end{array}$ & & & CIS (OCB -) & 9 & 1.2 & Without medication \\
\hline $21^{*}$ & --- & --- & --- & --- & --- & --- & --- & --- & --- \\
\hline 22 & 6 & $\mathrm{M}$ & $\begin{array}{l}\text { Cerebellar and } \\
\text { brainstem }\end{array}$ & & & CIS $(\mathrm{OCB}+)$ & 7 & 1.9 & Without medication \\
\hline 23 & 14 & $\mathrm{~F}$ & Sensory & & & $\mathrm{MS}(\mathrm{OCB}+)$ & 14 & 0.7 & Interferon $\beta$ 1A $44 \mu \mathrm{g}$ \\
\hline $24 *$ & --- & --- & --- & --- & --- & --- & --- & --- & --- \\
\hline 25 & 4 & M & Pyramidal & & & $\begin{array}{l}\text { CIS (OCB - / } \\
\text { AQP4-IgG -) }\end{array}$ & 5 & 0.3 & Without medication \\
\hline 26 & 5 & $\mathrm{~F}$ & $\begin{array}{l}\text { Pyramidal, cerebral and } \\
\text { visual }\end{array}$ & d Visual & & $\begin{array}{l}\text { CIS (OCB - / } \\
\text { AQP4-IgG -) }\end{array}$ & 10 & 5.6 & Antiepileptic drug \\
\hline 27 & 11 & $\mathrm{~F}$ & $\begin{array}{l}\text { Pyramidal, sensory, } \\
\text { visual, bowel and } \\
\text { bladder }\end{array}$ & & & $\begin{array}{l}\text { NMO (OCB - } \\
\text { /AQP4-IgG -) }\end{array}$ & 11 & 0.3 & Azatioprine \\
\hline
\end{tabular}

* Did not meet study inclusion criteria ** Excluded from analysis because images did not meet quality standards. 
After revising all the exams, we selected three of each patient while obeying the following criteria: (a) the first MRI (corresponding to each patient's first clinical episode); (b) a representative follow-up exam according to the evolution of each patient (exams of subsequent acute episodes in patients that had multiple episodes or simply follow-up exams in patients with a stable clinical course); and (c) the last exam of each patient at the end of the study.

Exams of every single patient were compared to each other in order to verify signs of dissemination of lesions in space and over time, and the resolution of old ones.

MRI studies were obtained respecting each patient's clinical necessity, but also according the limits of the Brazilian public national health system and the financial situation of the patient and their caregivers (through private health insurances).

When possible, patients obeyed the following recommendations: imaging study during the acute episodes and three months after treatment; in the absence of signs of relapse, patients using immunomodulatory treatments or immunosuppression would repeat the MRI every 6 months during the first year of follow-up and then yearly, afterwards; patients not using any drugs would repeat the MRI annually after resolution of acute episode.

(1) Statistical analysis

A descriptive analysis of continuous variable was made, using averages and standard deviations. Frequencies were calculated for categorical variables.

(2) Ethical aspects

It was approved by the local ethics committee and all patients agreed to participation in the study, having signed a consent agreement.

\section{Results}

\subsection{Patient Profile}

Among analyzed patients, follow-up duration at our clinic ranged from 2 to 95 months (mean 36.3 months). Twelve (55\%) were females. Mean age at first acute demyelination episode was 7.7 years old (ranging from 1 to 14 years old), with 16 (70\%) being under 10 years of age.

Predominant clinical manifestations in the first acute episode, according to the neurological function affected (using EDSS), were pyramidal tract involvement in 12 (52\%), brainstem involvement in 7 $(30 \%)$, encephalopathy symptoms in $6(26 \%)$ and cerebellar involvement in $5(22 \%)$. Other, less common symptoms included visual, vesical, intestinal and sensory disturbances.

The most common diagnosis was CIS in 8 patients (35\%), followed by MS in $7(30 \%)$ and NMO and ADEM, present in 4 patients, each (17\%).

Considering the whole group of patients, the mean time elapsed between the first acute episode and ADD diagnosis was 1.8 years (ranging from 3 months to 8 years). This period was generally shorter in patients with MS (mean duration of 1 year for patients under 10 years old and 0.7 year for those above) and ADEM (mean 1.0 year). Patients with CIS had a mean time until diagnosis of 1.8 years, while the patients with NMO took longer between the first acute episode and the diagnosis and commencement of immunosuppression (mean 4.5 years).

OCB in MS patients was positive in 5 of the 6 tested patients $(83 \%)$. In contrast, CIS patients had positive OCB in only 3 of the 7 tested patients (43\%). No patient with ADEM was tested for OCB and the only NMO patient tested was negative.

The AQP4-IgG was positive in only one (25\%) of the NMO patients, while no patient was with ADEM, EM or CIS tested positive.

Regarding treatment, all patients with a diagnosis of MS were, at the end of the study, using Interferon $\beta$ 1A. Two patients with NMO were using glatiramer acetate, one was using azathioprine and the last was using azathioprine in association with mycophenolate mofetil. One patient, with multiphasic ADEM, was using mycophenolate mofetil at the end of the study while the other ADEM patients as well as the CIS 
patients were not receiving any immunomodulatory or immunosuppressive medication at the end of the study.

\subsection{MRI Evaluation}

A total of 139 MRI exams were revised. Each patient underwent 2 to 15 imaging studies (mean: 6).
Fig. 1 summarizes the most relevant data for the radiological characterization of the patients.

The first MRI revealed that ADEM patients had more asymmetrical and bigger (over 1 or 2 centimeters wide), and some had tumefactive lesions, with contrast enhancement. Areas most often affected were the brain (periventricular, juxtacortical, infratentorial

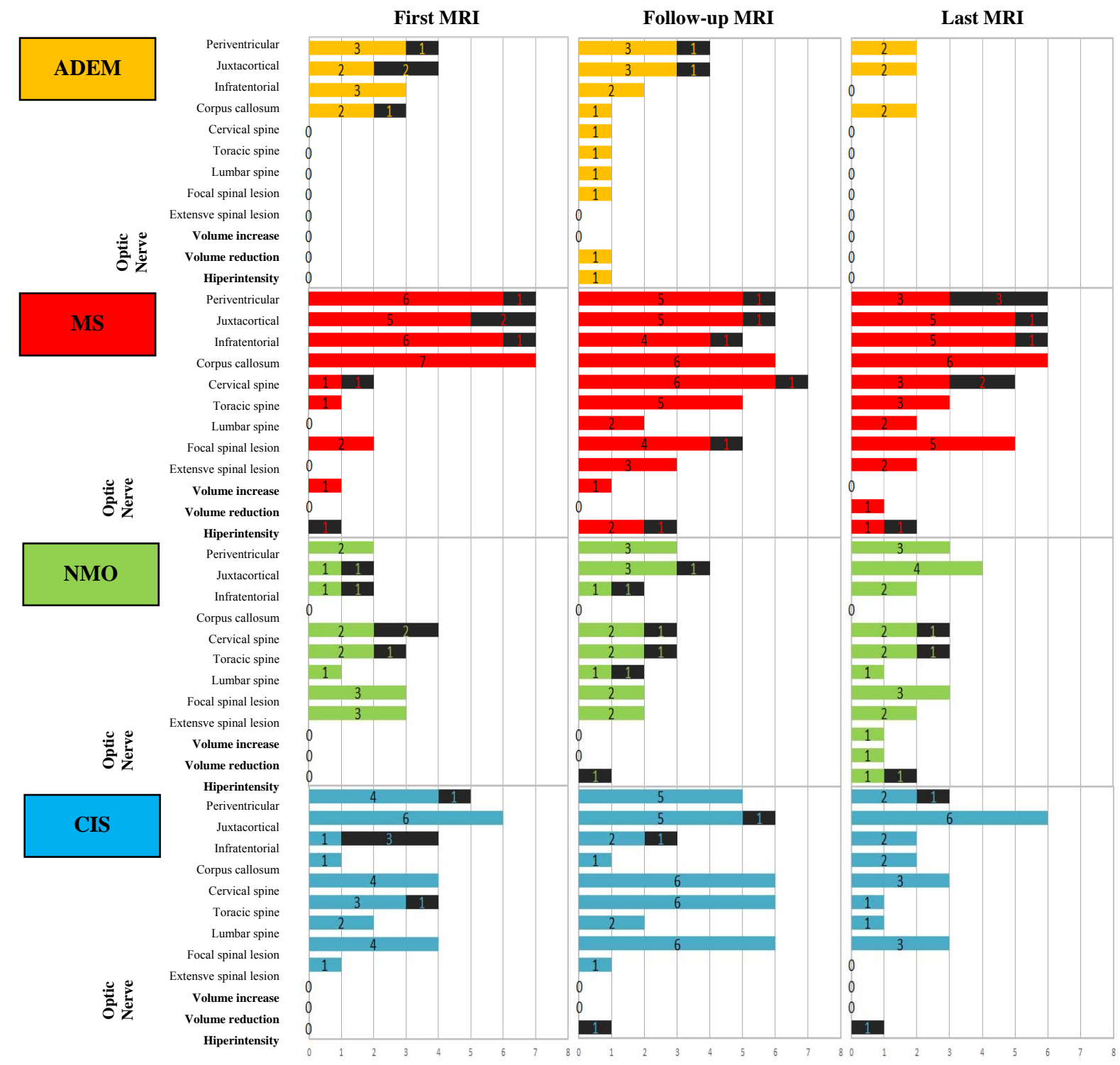

Fig. 1 MRI characteristics in patients with demyelinating disorders.

First column: radiological characteristics from each patient's first MRI; second column: follow-up MRI (most representative image of each patient's disease) and third column: last MRI at the end of the study. Each line in the graphic represents one of the CNS areas usually afflicted by demyelinating disorders. Bars represent number of patients affected. Black bars represent demyelinating lesions with contrast enhancement.

ADEM: acute disseminated encephalomyelitis; CIS: clinically isolated syndrome; MS: multiple sclerosis; NMO: neuromyelitis optica. 
and in the corpus callosum). The follow-up MRI generally demonstrated total or subtotal resolution of the lesions, with the exception of two patients: one with a diagnosis of multiphasic ADEM (who presented with new lesions in the medulla) and one who evolved with focal lesions in all three segments of the spinal cord. In the last exam, no patient had active lesions with contrast enhancement.

Patients with MS had smaller lesions (up to $1 \mathrm{~cm}$ ), localized mainly in the brain (periventricular, juxtacortical, infratentorial and corpus callosum) (Fig. 2A). Four patients with MS showed optic nerve alterations. All seven patients had focal spinal lesions (Fig. 2B), with mostly cervical involvement, while four patients had longitudinally extensive transverse myelitis (LETM; with an extension equal to or greater than 3 vertebral bodies) during their time in the study. All MS patients had contrast enhanced lesions during their disease, and four of them still showed contrast enhancement in their last exam, despite treatment.

The four NMO patients had LETM, while three had it in their first acute episode. Two patients had ON (optic neuritis): one with unilateral involvement and another bilateral. Hyperintense lesions, volume alterations and contrast enhancement were observed (Fig. 3).

Only one CIS patient had some optic nerve alteration, that being hyperintense signal change. The seven CIS patients that had a spine MRI done showed focal lesions, with a predominant cervical and thoracic involvement, and one patient had LETM. These patients' last exams showed radiological improvement and none of them had contrast enhanced lesions.

\section{Discussion}

This study summarizes the data of a pediatric ADD cohort followed in a single specialized medical institution using a uniformed approach, while still tailored to each patient's specific needs and clinical conditions.

Selection bias explains the low incidence of ADEM in this cohort: our center is a high-complexity terciary care facility and patients were referred by their primary care physicians. Hence, the ADEM cases referred were either those who presented with more than one acute event or those who did not obtain success after the initial round of therapy.

Fragoso et al. [9] when evaluating Brazilian MS patients, described that there could be a delay of over 10 years until patients under 10 years old are diagnosed, when compared to older patients. In our study, time elapsed until diagnosis was, in average, of one year for MS patients. Patients with ADD in general were diagnosed, in our cohort, in 1.8 years in average. That time was longer for NMO patients (mean: 4.5 years).

Clinically the most common MS presentation in children is polyfocal, contrary to what is observed in adults, where presentation is usually monofocal [10]. Curiously, but in accordance to what was observed in an Indian cohort, our younger (under 10 years old) patients had a majority of monofocal presentations [11].

The most frequent symptoms reported in pediatric MS are related to the motor and brainstem dysfunctions, followed by sensory symptoms and ataxia [12, 13]. But in our study, the most frequent symptoms were related to the brainstem and cerebellum.

OCB positivity is not obligatory for an MS diagnosis [8], especially because it can be negative in a first acute episode of demyelination. Nevertheless, the presence of OCB can aid the diagnosis whenever it is an important diagnostic hypothesis. In this cohort only one MS patient out of the 6 that were tested (17\%) had a negative OCB result. Furthermore, three (43\%) of the CIS patients that were tested had positive OCB, despite which they still did not fulfill the MS diagnostic criteria.

The AQP4-IgG is an important NMO disease biomarker because of its high specificity [14], but a negative result doesn't exclude the diagnosis. Banwell 


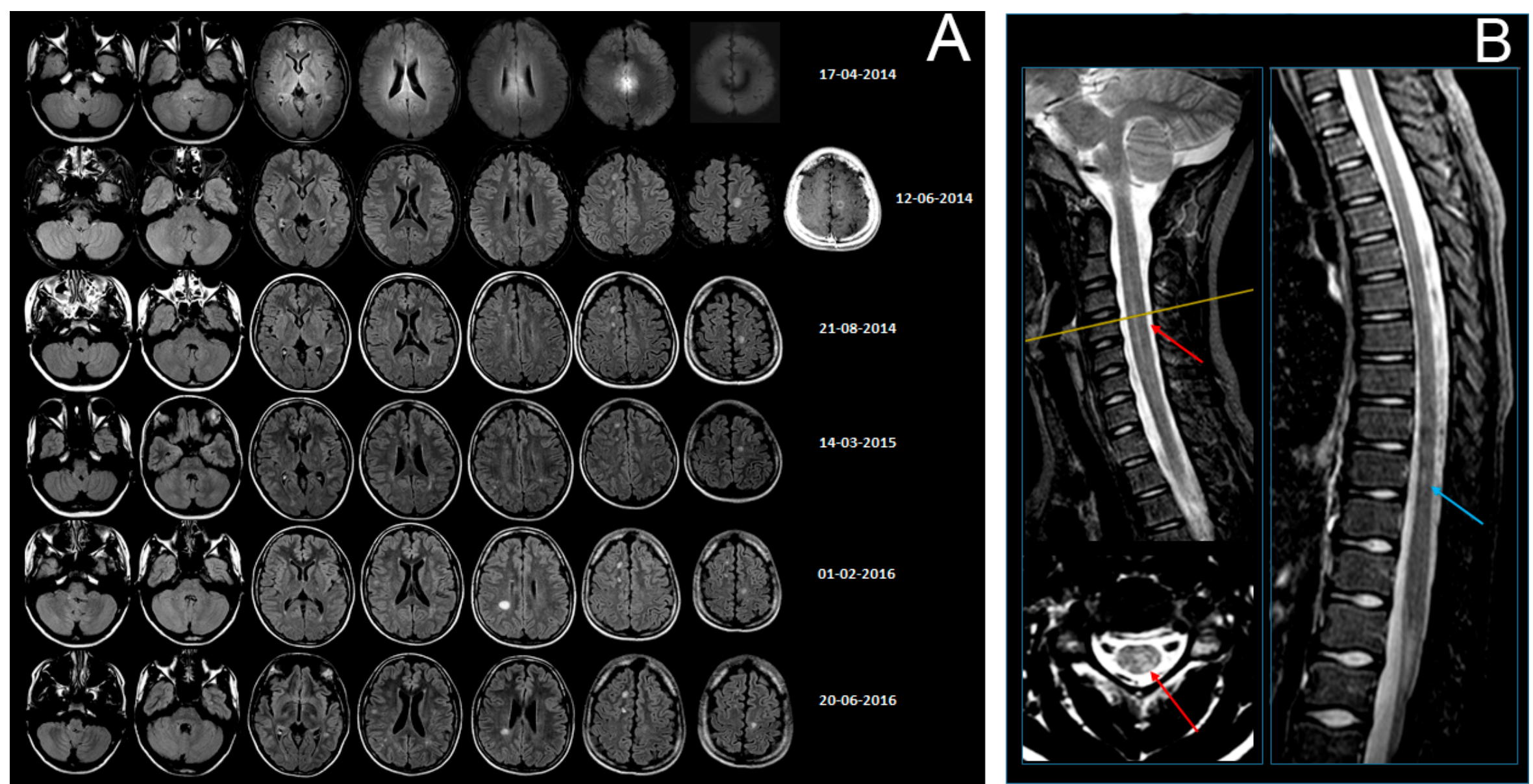

Fig. 2 MS patient MRI. (A) FLAIR Brain MRI. Evolution in time and space of the demyelinating lesions; (B) T2-weighted cervical and thoracic spinal cord MRI. Small cervical spine and thoracic lesions. 


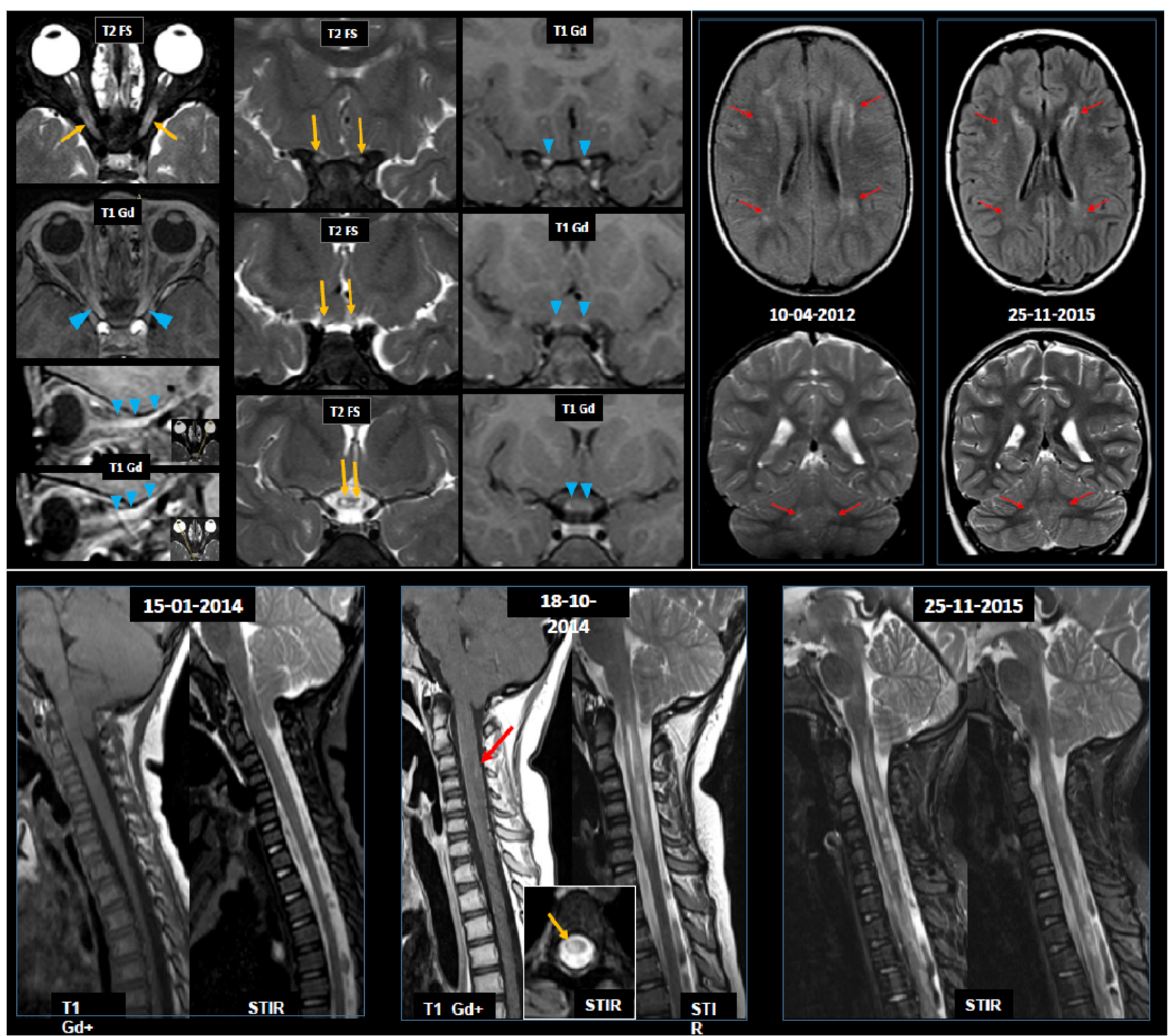

Fig. 3 NMO patient MRI.

(A) Orbits MRI (April/2012). Hyperintense signal in the T2-weighted image with fat suppression, and contrast enhancement of the nerves and optic chiasm, accompanied by increased volume of these structures. (B) Brain MRI (April/2012 and November/2015). Various white matter lesions (periventricular, subcortical and infratentorial) without significant change are in the follow-up exam or contrast enhancement. (C) Cervical and higher thoracic segments spinal cord MRI (left) January/2014 - Spine with preserved volume, without contrast enhanced lesions. Chiari malformation, type I, is evident, with cerebellar tonsils below the foramen magnum. (Center) October/2014-Marked involvement of the cervical spine, with volume increase and longitudinally extensive lesions (extension surpasses 3 vertebrae), and subtle foci of contrast enhancement (red arrow). The thoracic spinal cord also shows foci of hyperintense signal (orange arrow, on the level of T5), without contrast enhancement. Subtle insinuation of the cerebellar tonsils through the foramen magnum: less prominent Chiari malformation, type 1. (Right) November/2015-More extensive involvement of the cervical and thoracic spinal cord, maintains the pattern of nearly continuous longitudinally extensive lesions.

et al. [15] found a 47\% AQP4-IgG positivity in pediatric NMO patients, and a $78 \%$ positivity rate when evaluating exclusively the population with relapsing NMO. In this study, out of the four NMO patients, only one presented with a positive
AQP4-IgG titer. That may be because of our small sample size or because of the varied quality of assay kits available in different Brazilian laboratories, especially considering reports of the occurrence of false negative results in up to $30 \%$ of the cases [6]. 
Treatment of ADD in Brazil depends highly on which medications are freely available for the patients in the public national health system (SUS). The ones available, at the time of this publication, are the interferon $\beta 1 \mathrm{~A}$ and glatiramer acetate [16]. All our pediatric MS patients are currently using interferon $\beta$ $1 \mathrm{~A}$, and only one of them has had a clinical relapse, attributed to the irregular use of the medication. One NMO patient had a relapse and had to have the regular therapeutic scheme modified to azathioprine associated to oral corticosteroid therapy, which was progressively substituted by mycophenolate mofetil.

(1) ADEM

One ADEM patient had to stay in prolonged oral corticosteroid therapy, due to the occurrence of relapses on weaning attempts. In this patient, because of the diagnosis of multiphasic ADEM, mycophenolate mofetil was started and was successful in allowing the suspension of oral corticosteroids without new relapses.

ADEM can affect any area of the CNS and each patient usually has lesions of varied sizes. These typically affect the central and subcortical brain white matter, as well as the U-fibers, cerebellum, brainstem and spinal cord [17]. This very pattern has been observed in our patients.

As for the presence of focal lesions, it is usually dispersed along the three segments of the spinal cord, only one of four patients presented it. Other authors had already reported an incidence of spinal lesions in up to 1 in every 3 ADEM patients, with those lesions usually being large, confluent and affecting multiple spinal segments [18].

Regarding treatment results, most patients in our cohort had a partial or complete resolution of the radiological abnormalities, which is a similar finding to the ones reported [19].

(2) Pediatric MS

All patients with MS had, on their first radiological exam, multiple encephalic lesions, localized in the periventricular, juxtacortical and infratentorial white matter and in the corpus callosum, with three patients exhibiting contrast enhancement in their lesions. Unfortunately, definite MS diagnosis could only be reached after new clinical episodes in five patients and after appearance of new, clinically silent lesions in the follow-up MRI of two patients, hence fulfilling the time and space dissemination MS diagnostic criteria [8].

It is a known fact that lesions can disappear in the beginning of the pediatric disease, while it is more common that old lesions remain unchanged in the following exams in the adult MS population [20]. In our cohort, the resolution of lesions during the patients' evolution was observed as well.

Despite not being common in MS, the LETM can happen in up to $10 \%$ of pediatric MS patients [15]. In our cohort, all MS patients had spine lesions, four of which had LETM, a much higher incidence than previously reported.

(3) NMO

All NMO patients had at least one episode of LETM and two had neuritis optica, while only one had positive AQP4-IgG. This situation is in accordance with the revised diagnostic criteria for NMO in adults, which includes the patients with the NMOSD (NMO spectrum disorders) and the patients with negative AQP4-IgG, as long as they fulfill the minimum requirements: at least two typical presentations, one being an episode of transverse myelitis, neuritis optica or area postrema syndrome; dissemination in space and the exclusion of other possible diagnoses [21].

Cerebral lesions with supratentorial predominance, usually big and isolated and with mass effects were observed in three NMO patients in this cohort. Other authors had already reported this type of lesion in NMO patients, in the supratentorial area as well as in the brainstem [7]. These lesions, even though they might suggest NMOSD, when unaccompanied by more typical NMO lesions, cannot be adequately differentiated from typical MS lesions, particularly in 
AQP4-IgG negative patients [21].

The radiological evolution of the cohort's patients shows a predominance of spinal lesions in NMO, including LETM, while in MS there was preponderance of encephalic lesions, while spinal lesions were mostly focal. This finding supports the fact that NMO is a distinct inflammatory CNS disease from MS [21].

\section{(4) CIS}

The patients in this cohort that were diagnosed with CIS had generally, a single clinical episode, which did not fulfill the diagnostic criteria for ADEM (neuritis optica or transverse myelitis) nor did they fulfill the imaging criteria for MS or NMO. The radiological follow-up demonstrated shrinking or resolution of the lesions. Verhey et al. [22] in 2011, following 322 children with pediatric ADD, observed that those with a single non-ADEM event and with normal brain MRI, as is the case with neuritis optica or transverse myelitis, carry a very low risk $(<3 \%)$ of an MS diagnosis.

\section{Conclusions}

The ADEM and CIS patients' MRI showed an important reduction of the lesions, as opposite to MS and NMO patients. Early diagnosis is possible, even if challenging, for these are rare conditions that require specialized professionals and complementary exams not always easily available in the Brazilian public health system. This study shows that these diseases can occur in the pediatric population, requiring from the pediatrician and pediatric neurologist a guided diagnostic suspicion.

It is of major importance to observe that the longitudinal follow-up of the patients in a single specialized medical institution and the use of a specific diagnosis treatment and diagnosis protocol can have positive effects over the speed of correct diagnosis and timely treatment, with the objective of minimizing these patients' sequelae and preventing new demyelinating events.
Still, the fact that the MRI exams in this study were performed in different machines and following various neuroimaging acquisition techniques and protocols did not allow for a more standardized and detailed evaluation of the demyelinating lesions, hindering the precise evaluation of the number and spread of lesions and their volumes.

The MRI is an important tool in the monitoring of activity in the ADD, and that is why we hope that a future study following a specific protocol for the acquisition of images in ADD patients may aid in establishing a clearer clinical-radiological-laboratorial correlation, shortening the time between the acute demyelinating event and the treatment, improving the prognostic of ADD patients. Another benefit of having a specific imaging protocol might involve the shorter exam duration, leading to a shorter anesthesia time.

\section{References}

[1] Sudhakar, S. V., Muthusamy, K., Mani, S., Gibikote, S., and Shroff, M. 2015. "Imaging in Pediatric Demyelinating and Inflammatory Diseases of the Brain-Part 1." Indian J. Pediatr. 83 (9): 952-64. doi:10.1007/s12098-015-1916-y.

[2] Noseworthy, J. H., Lucchinetti, C., Rodriguez, M., and Weinshenker, B. G. 2000. "Multiple Sclerosis." N. Engl. J. $\quad$ Med. $\quad 343 \quad$ (13): $\quad 938-52$. doi:10.1056/NEJM200009283431307.

[3] Gadoth, N. 2003. "Multiple Sclerosis in Children." Brain Dev. $25 \quad$ (4): 229-32. doi:10.1016/S0387-7604(03)00035-4.

[4] Boiko, A., Vorobeychik, G., Paty, D., Devonshire, V., and Sadovnick, D. 2002. "Early Onset Multiple Sclerosis: A Longitudinal Study.” Neurology 59 (7): 1006-10.

[5] Verhey, L. H., Narayanan, S., and Banwell, B. 2013. "Standardized Magnetic Resonance Imaging Acquisition and Reporting in Pediatric Multiple Sclerosis." Neuroimaging Clin. N. Am. 23 (2): 217-26. doi:10.1016/j.nic.2012.12.003.

[6] Krupp, L. B., Banwell, B., and Tenembaum, S. 2007. "Consensus Definitions Proposed for Pediatric Multiple Sclerosis and Related Disorders." Neurology 68 (16 SUPPL 2): 7-12. doi:10.1212/01.wnl.0000259422.44235.a8.

[7] Krupp, L. B., Tardieu, M., Amato, M. P., et al. 2013. "International Pediatric Multiple Sclerosis Study Group Criteria for Pediatric Multiple Sclerosis and 
Immune-Mediated Central Nervous System Demyelinating Disorders: Revisions to the 2007 Definitions." Mult. Scler. J. 19 (10): 1261-7. doi: $10.1177 / 1352458513484547$.

[8] Polman, C. H., Reingold, S. C., Banwell, B., et al. 2011. "Diagnostic Criteria for Multiple Sclerosis: 2010 Revisions to the McDonald Criteria." Ann Neurol. 69 (2): 292-302. doi:10.1002/ana.22366.

[9] Fragoso, Y. D., Brooks, J. B. B., and Leal, T. M. D. S. 2012. "Perfil descritivo de esclerose múltipla com início até os 16 anos nos pacientes de um centro de referência do estado de São Paulo." Rev. Paul. Pediatr. 30 (4): 617-20. doi:10.1590/S0103-05822012000400023.

[10] Yeh, E. A., Chitnis, T., Krupp, L., et al. 2009. "Pediatric Multiple Sclerosis." Nat. Publ. Gr. 5 (11): 621-31. doi:10.1038/nrneurol.2009.158.

[11] Gulati, S., Chakrabarty, B., Kumar, A., Jain, P., Patel, H., and Saini, L. 2015. "Acquired Demyelinating Disorders of Central Nervous System: A Pediatric Cohort." Ann. Indian Acad. Neurol. $18 \quad$ (5): 48. doi:10.4103/0972-2327.164829.

[12] Banwell, B., Ghezzi, A., Bar-Or, A., Mikaeloff, Y., and Tardieu, M. 2007. "Multiple Sclerosis in Children: Clinical Diagnosis, Therapeutic Strategies, and Future Directions." Lancet Neurol. 6 (10): 887-902. doi:10.1016/S1474-4422(07)70242-9.

[13] Mikaeloff, Y., Suissa, S., Vallée, L., et al. 2004. "First Episode of Acute CNS Inflammatory Demyelination in Childhood: Prognostic Factors for Multiple Sclerosis and Disability." J. $\quad$ Pediatr. $144 \quad$ (2): 246-52. doi:10.1016/j.jpeds.2003.10.056.

[14] Lennon, V. A., Wingerchuk, D. M., Kryzer, T. J., et al. 2004. "A Serum Autoantibody Marker of Neuromyelitis
Optica: Distinction from Multiple Sclerosis." Lancet (London, England). $364 \quad$ (9451): 2106-12. doi:10.1016/S0140-6736(04)17551-X.

[15] Banwell, B., Tenembaum, S., Lennon, V. A., et al. 2008. "Neuromyelitis Optica-Igg in Childhood Inflammatory Demyelinating CNS Disorders." Neurology. 70 (5): 344-52. doi:10.1212/01.wnl.0000284600.80782.d5.

[16] Ministério da Saúde. Portaria N. 199, de 30 de Janeiro de 2014.

[17] Pohl, D., Alper, G., Van Haren, K., et al. 2016. "Acute Disseminated Encephalomyelitis." Neurology. 87 (2): S38-45. doi:10.1136/bmj.1.5029.1246.

[18] Tenembaum, S., Chamoles, N., and Fejerman, N. 2002. "Acute Disseminated Encephalomyelitis: A Long-Term Follow-up Study of 84 Pediatric Patients." Neurology 59 (1245): 1224-31. doi:10.1212/wnl.59.8.1224.

[19] Tenembaum, S., Chitnis, T., Ness, J., and Hahn, J. S. 2007. "Acute Disseminated Encephalomyelitis." Neurology. doi:10.1212/01.wnl.0000259404.51352.7f.

[20] Chabas, D., Castillo-Trivino, T., Mowry, E. M. Strober, J. B., Glenn, O. A., Waubant, E., and Vanishing, M. S. 2008. "T2-Bright Lesions before Puberty: A Distinct MRI Phenotype?" Neurology 71 (14): 1090-3. doi:10.1212/01.wnl.0000326896.66714.ae.

[21] Wingerchuk, D. M., Banwell, B., Bennett, J. L., et al. 2015. "International Consensus Diagnostic Criteria for Neuromyelitis Optica Spectrum Disorders.” Neurology 85 (2): 177-89. doi:10.1212/WNL.0000000000001729.

[22] Verhey, L. H., Branson, H. M., Shroff, M. M., et al. 2011. "MRI Parameters for Prediction of Multiple Sclerosis Diagnosis in Children with Acute CNS Demyelination: A Prospective National Cohort Study." Lancet Neurol. 10 (12): 1065-73. doi:10.1016/S1474-4422(11)70250-2. 